\title{
$5,-05$ 135200
}

DESIGN OF HIGH SPEED PROPROTORS USING

N93-18角65 MULTIOBJECTIVE OPTIMIZATION TECHNIQUES

\author{
by \\ Thomas R. McCarthy \\ and
}

Aditi Chattopadhyay

Department of Aerospace and Mechanical Engineering

Arizona State University

Tempe, Arizona 85287 - 6106

Proposed Abstract for

1993 AIAA/AHS/ASEE Aerospace Design Conference

February 15-18, 1993

Irvine, California 


\title{
Design of High Speed Proprotors Using Multiobjective Optimization Techniques
}

\author{
by \\ Thomas R. McCarthy ${ }^{\dagger}$ \\ and \\ Aditi Chattopadhyay ${ }^{\dagger}$ \\ Department of Aerospace and Mechanical Engineering \\ Arizona State University \\ Tempe, Arizona
}

\section{Introduction}

Over the last few years, there has been a revival of interest in VTOL aircraft capable of operating in fixed wing as well as rotary wing mode. High speed rotorcraft designs, such as the tilting rotor configuration, pose an entirely new problem in the rotary wing field. The design goals for this class of aircraft include low downwash velocity in hover, good low speed maneuverability and cruise speeds of $350-500$ knots ${ }^{1}$. Several new concepts $^{2-5}$ have recently been proposed to meet these design goals. Extensive research performed in this field have led to the XV-15 research aircraft and ultimately to the production of the V-22 Osprey tilting rotor for the US Navy.

The combined requirements of efficient high speed performance of a fixed wing aircraft and good helicopter-like hover characteristics complicates the design process of tilting high speed proprotor aircraft. It is necessary to maintain good aerodynamic efficiency in high speed axial flight without degrading hover efficiency. This often leads to conflicting design requirements. For example, improved efficiency in high speed

$\dagger$ Graduate Research Assistant, Member AIAA, AHS

t† Associate Professor, Member AIAA, AHS, ASME 
cruise demands high drag divergence Mach numbers which are normally associated with thin airfoils. This however, reduces the hover figure of merit by reducing $C_{T} / \sigma$. Therefore, to maintain the required thrust ceiling in hover, the rotor solidity has to increase. Also as the forward speed increases, helical tip Mach number limitations caused by whirl flutter, require a reduction in the rotor rotational velocity. Introducing blade sweep can alleviate this problem by reducing the effective chordwise Mach number, which allows for higher speeds, without reducing the rotor RPM. Therefore the proper design of proprotor blades capable of achieving the design objectives must consider the right combination of airfoil thickness and blade sweep in addition to other aerodynamic variables such as planform and twist.

Several studies have been performed ${ }^{6-9}$ to study design trade offs between the two flight modes. For example, Johnson et al. ${ }^{6}$ performed a detailed study on the performance, maneuverability and stability of high speed tilting proprotor aircraft, including the XV-15 and V-22. Liu and McVeigh ${ }^{7}$ recently studied the use of highly swept rotor blades for high speed tilt rotor use. However, formal optimization techniques were not used in these studies. Recently an effort was initiated by Chattopadhyay and Narayan 8,9 to develop formal multidisciplinary optimization procedures for the design of civil high speed tilting proprotor blades. The propulsive efficiency in axial flight was maximized with constraints on the figure of merit in hover, aeroelastic stability in cruise and other aerodynamic and structural design criteria. The purpose of the present paper is to formulate the optimum design problem of high speed proprotors using multiobjective optimization techniques with the integration of the necessary disciplines.

\section{Problem Definition}

\footnotetext{
A integrated, multiobjective optimization procedure is developed for the design of high speed proprotors with the coupling of aerodynamic, dynamic, aeroelastic and
}

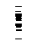


structural criteria. The objectives are to maximize propulsive efficiency in high speed cruise and rotor figure of merit in hover. Constraints are imposed on rotor blade aeroelastic stability in cruise and on total blade weight. Two different multiobjective formulation procedures, the Min $\Sigma \beta$ and the K-S function approaches are used to formulate the two-objective optimization problem.

\section{Aerodynamic Model}

The rotor studied is a wind tunnel model an existing advanced technology proprotor, which is a three bladed rotor with a rigid hub. Cubic variations are assumed for the chord (c) and twist $(\theta)$ distributions to model the blade aerodynamics,

$$
\begin{aligned}
& c(\bar{y})=c_{0}+c_{1}(\bar{y}-0.75)+c_{2}(\bar{y}-0.75)^{2}+c_{3}(\bar{y}-0.75)^{3} \\
& \theta(\bar{y})=\theta_{0}+\theta_{1}(\bar{y}-0.75)+\theta_{2}(\bar{y}-0.75)^{2}+\theta_{3}(\bar{y}-0.75)^{3}
\end{aligned}
$$

where $\bar{y}$ denotes the nondimensional blade radius. Note that $c_{0}$ represents the chord and $\theta_{0}$ the twist at the 75 percent blade radius, respectively. A quadratic lifting line is used and is defined as follows.

$$
x=f(y)=\varepsilon_{1 y}+\varepsilon_{2} y^{2}
$$

where $\varepsilon_{1}, \varepsilon_{2}$ are constants that determine the curvature, and are defined such that

$$
\left|\varepsilon_{\mathrm{i}}\right| \leq \zeta_{\mathrm{i}}
$$

where $\zeta_{\mathrm{i}}(\mathrm{i}=1,2)$ are prescribed bound for the curvature parameters. These bounds allow for either forward or backward in-plane curvature. When $\varepsilon_{1}$ and $\varepsilon_{2}$ are equal to zero the lifting line will be a straight line. The blade sweep, based upon this lifting line distribution, assumes the following form 


$$
\begin{aligned}
\Lambda(\bar{y}) & =\frac{180}{\pi} \tan ^{-1}\left(\frac{\mathrm{dx}}{\mathrm{dy}}\right) \\
& =\frac{180}{\pi} \tan ^{-1}\left(\varepsilon_{1}+2 \varepsilon_{2} \bar{y}\right)
\end{aligned}
$$

where $\Lambda(\bar{y})$ is the sweep distribution, in degrees, defined to be positive aft of the straight lifting line.

\section{Structural Model}

The structural model used for the problem consists of a simple two-celled box beam as shown in Fig. 1. The beam is considered to be the principal load carrying member of the proprotor and the stiffness contributions from the honeycomb and the nonstructural or tuning weights are placed at the blade tip and are distributed along the planform. The total blade weight comprises the weight of the box beam, the skin, the honeycomb and the nonstructural weights. The wall thicknesses of the box beam are assumed to vary in proportion to the chord distribution.

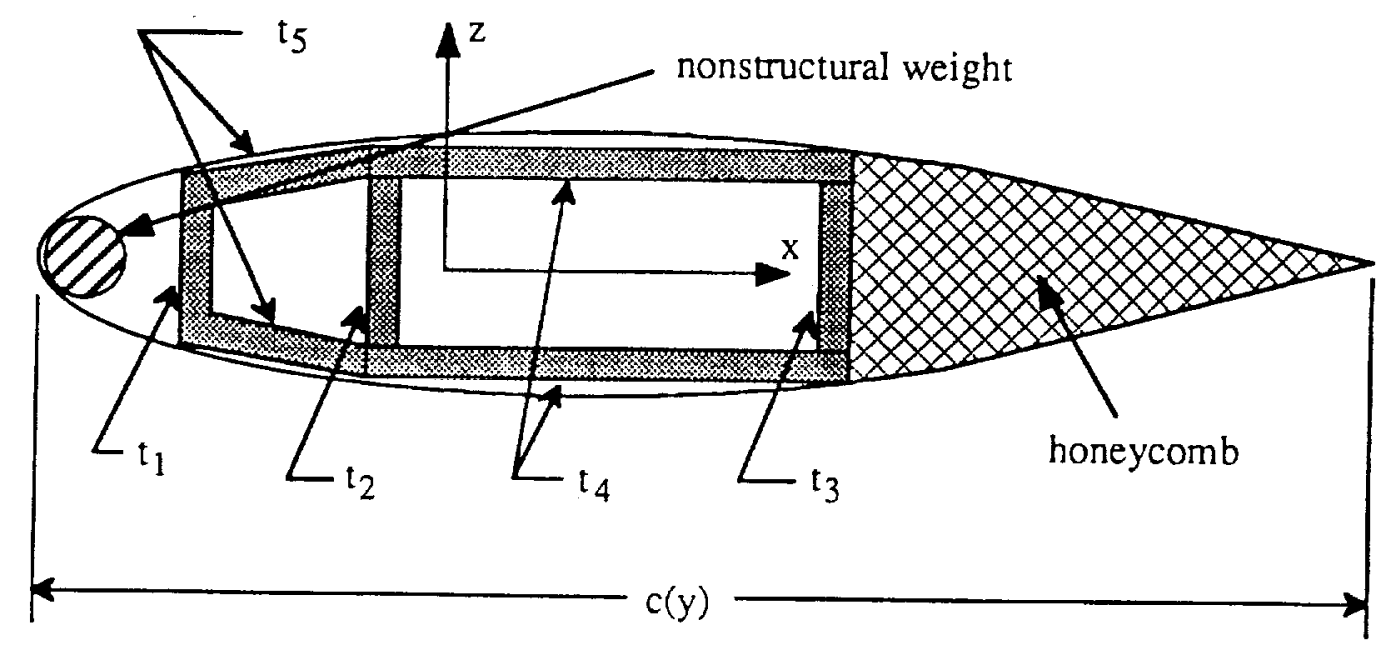

Figure 1 Double-celled box beam configuration 


\section{Optimization}

Objective Functions: The multiobjective optimization procedure is used to simultaneously maximize the rotor propulsive efficiency in high speed cruise and the hover figure of merit.

Design Variables: Both aerodynamic and structural design variables are used. The aerodynamic design variables include chord, twist and sweep distribution coefficients (Eqns. 1, 2 and 5). The structural design variables comprise roots values of the wall thicknesses of the two-cell box beam and the magnitudes of the nonstructural weights, at the tip, distributed spanwise.

Constraints: To avoid the possible occurrences of air and/or ground resonance associated with a soft inplane rotor, it is important to maintain the value of the lowest natural frequency in hover, $f_{1}$, above $1 / \mathrm{rev}$. Therefore the following constraint is imposed.

(i) $\mathrm{f}_{1}>1 / \mathrm{rev}$

It is important to impose aeroelastic stability constraints to prevent any degradation of the rotor stability in high speed cruise. This is all the more important when the blade mass and stiffness are altered during optimization. The stability constraints are expressed as follows.

(ii) $\alpha_{k} \leq-v_{k} \quad k=1,2, \ldots, K$

Where $\mathrm{K}$ represents the total number of modes considered, and $\alpha_{k}$ is the real part of the stability root. The quantity $v_{\mathrm{k}}$ denotes the minimum allowable blade damping and is defined to be a small positive number. To avoid incorporation of weight penalties, after optimization, the total blade weight is constrained as follows.

(iv) $\mathrm{W} \leq \mathrm{W}_{\mathrm{U}}$ 


\section{Multiobjective Optimization}

A typical optimization problem involving multiple objective functions can be mathematically posed as follows.

$$
\text { Minimize } \quad \mathrm{F}_{\mathrm{k}}\left(\phi_{\mathrm{n}}\right) \quad \begin{aligned}
& \mathrm{k} \\
& \quad \mathrm{n}=1,2, \ldots, \mathrm{NOBJ} \quad \text { (objective functions) } \\
& \mathrm{n}=1,2, \ldots, \mathrm{NDV}
\end{aligned}
$$

Subject to

$$
\mathrm{g}_{\mathrm{j}}\left(\phi_{\mathrm{n}}\right) \leq 0 \quad \mathrm{j}=1,2, \ldots, \mathrm{NCON} \quad \text { (inequality }
$$

constraints)

$$
\phi_{n_{L}} \leq \phi_{n} \leq \phi_{n_{U}}
$$

(side constraints)

where NOBJ denotes the number of objective functions, NDV is the number of design variables and NCON is the total number of constraints. The subscripts $L$ and $U$ denote lower and upper bounds, respectively, on the design variable $\phi_{n}$. A description of the multicriteria design objective formulation follows.

This study examines three multiobjective function formulation techniques that are less judgmental than the Pareto based weighting factors and are therefore more suited to large scale, highly nonlinear optimization problems that are associated with rotary wing design. The two multiobjective function techniques used are the Minimum Sum Beta (Min $\Sigma \beta$ ) and the Kreisselmeier-Steinhauser (K-S) function approaches. A description of these methods follows.

Minimum Sum Beta (Min $\Sigma \beta$ ): This method was first used by Weller at al. ${ }^{10}$ to formulate a two objective function rotor vibration problem. Using these technique, pseudo-design variables that represent tolerances of the individual objective functions from prescribed tolerances are introduced. The objective function, $\widetilde{\mathrm{F}}_{1}(\Phi)$, is then defined 
as a linear combination of these tolerances of each objective function to their specified target values as follows

$$
\tilde{F}_{1}(\Phi)=\sum_{k=1}^{N O B J} \beta_{k}
$$

where $\beta_{\mathrm{k}}$ are pseudo design variables with properties such that the original objective functions $F_{k}$ remain within a $\beta_{k}$ tolerance of some prescribed values. This requirement introduces new constraints of the following form.

$$
\frac{F_{k}-\dot{F}_{k}}{\tilde{F}_{k}} \leq \beta_{k} k=1,2, \ldots, \text { NOBJ }
$$

The quantities $\bar{F}_{k}$ are the prescribed target values of the individual objective functions $F_{k}$. Using the above formulation, as the objective function, and correspondingly the values of $\beta_{k}$, are reduced to zero, the values of the individual objective functions $F_{k}$ are driven to their prescribed values, $\bar{F}_{\mathbf{k}}$. The design variables for the Min $\Sigma \beta$ formulation comprise the original set of design variables and the pseudo design variables, $\beta_{k}$.

Kreisselmeier-Steinhauser (K-S) function: This technique was first utilized by Sobieski et al. ${ }^{11}$ at the NASA Langley Research Center. The first step in formulating the objective function in this approach involves transformation of the original objective functions into reduced objective functions ${ }^{12}$. These reduced objective functions take the form

$$
\mathrm{F}_{\mathrm{k}}^{*}(\Phi)=\frac{\mathrm{F}_{\mathrm{k}}(\Phi)}{\mathrm{F}_{\mathrm{k}_{0}}}-1.0-\mathrm{g}_{\max } \leq 0 \quad \mathrm{k}=1, \ldots, \mathrm{NOBJ}
$$

where $F_{k_{0}}$ represents the value of $F_{k}$ calculated at the beginning of each iteration. The quantity $g_{\max }$ is the value of the largest constraint corresponding to the design variable vector $\Phi$ and is held to be constant for each iteration. These reduced objective functions 
are analogous to the previous constraints, and therefore a new constraint vector $\mathrm{g}_{2 \mathrm{~m}}(\Phi)$ $(m=1,2, \ldots, M)$ is introduced, where again $M=N C O N+$ NOBJ. The new objective function to be minimized is then defined, using the K-S function as follows:

$$
\tilde{\mathrm{F}}_{2}(\Phi)=\mathrm{f}_{\max }+\frac{1}{\rho} \ln \sum_{\mathrm{m}=1}^{M} \mathrm{e}^{\rho\left(\mathrm{g}_{\mathrm{m}}(\phi)-\mathrm{f}_{\max }\right)}
$$

where $f_{\max }$ is the largest constraint corresponding to the new constraint vector, $g_{2 m}(\Phi)$, and in general is not equal to $g_{\max }$. The multiplier $\rho$ can be considered analogous to a draw-down factor where $\rho$ controls the distance from the surface of the K-S objective function to the surface of the maximum constraint function. When $\rho$ is large the K-S function will closely follow the surface of the largest constraint function. When $\rho$ is small, the K-S function will include contributions from all violated constraints. The design variable vector $\Phi$ is identical to that used in the Min $\Sigma \beta$ approach.

\section{Analysis}

Dynamic. Aerodynamic and Aeroelastic Analyses The aerodynamic, dynamic and aeroelastic analysis of the high speed proprotor is performed using the code CAMRAD/JA 13 . The code has the capability of analyzing both helicopter and tilting rotor aircraft. Wind tunnel trim options are used as the reference blade is a wind tunnel blade model. In cruise, the blade is trimmed to specific rotor lift and drag coefficients using the rotor collective and cyclic pitch angles. A prescribed wake model, as implemented in CAMRAD/JA, is used to model the aerodynamics in hover and the rotor is trimmed to a specific value of the coefficient of power. In axial flight, the components of the induced velocity are negligible compared to the high forward speed of the rotor. Therefore, uniform inflow conditions are used to model the aerodynamics in this case. 
The aeroelastic stability analysis in cruise is analyzed with a constant coefficient approximation.

Structural Analysis: The detailed structural analysis of the rotor blade is performed based upon the two-celled trapezoidal box beam using an inhouse code that was recently developed specifically for this application.

\section{Optimization Implementation}

The optimization is performed by using the program CONMIN 14 . The program uses the method of feasible directions to solve nonlinear constrained optimization problems. To reduce the computational effort, an approximate analysis technique is used to compute the objective function and constraint values during iterations within the optimizer. For this problem the two-point exponential hybrid approximation technique ${ }^{15}$ is used. This technique takes its name from the fact that the exponent used in the expansion is based upon gradient information from the previous design point. The technique is formulated as follows.

$$
\hat{F}(\Phi)=F\left(\Phi_{0}\right)+\sum_{n=1}^{N D V}\left[\left(\frac{\phi_{n}}{\phi_{o_{n}}}\right)^{p_{n}}-1.0\right] \frac{\phi_{o_{n}}}{p_{n}} \frac{\partial F\left(\Phi_{0}\right)}{\partial \phi_{n}}
$$


where

$$
p_{n}=\frac{\log \left\{\frac{\left(\frac{\partial F\left(\Phi_{1}\right)}{\partial \phi_{n}}\right)}{\left(\frac{\partial F\left(\Phi_{0}\right)}{\partial \phi_{n}}\right)}\right\}}{\log \left(\frac{\partial \phi_{1_{n}}}{\partial \phi_{o_{n}}}\right)}+1.0
$$

The quantity $\Phi_{1}$ refers to the design variable vector from the previous iteration and the quantity $\Phi_{0}$ denotes the current design vector. The exponent $\mathrm{p}_{\mathrm{n}}$ can be considered as a "goodness of fit" parameter, which explicitly determines the trade-offs between traditional and reciprocal Taylor series based expansions (also known as a hybrid approximation technique). Details of this method can be found in Ref. 15.

\section{Results and Discussions}

A wind tunnel model of an existing high speed proprotor is used as a baseline design. The optimization for this problem is performed with a cruise velocity of 400 knots and a rotational velocity of $\Omega=375 \mathrm{RPM}$ (tip speed of $491 \mathrm{ft} / \mathrm{s}$ ) in axial flight. The operating condition is 20,000 feet above sea level. In hover, a rotational velocity of $\Omega=570$ RPM (tip speed of $746 \mathrm{ft} / \mathrm{s}$ ) is used at sea-level conditions. The high forward flight speed of 400 knots represents the target cruise value for high-speed rotorcraft. The tip speed is reduced in the airplane mode so that the helical tip Mach number stays below $M_{d d}$ (the drag divergence Mach number). The rotor RPM in cruise (375) is selected after performing a parametric study on the effect of forward speed and rotor RPM on propulsive efficiency. A value of $\mathrm{C}_{\mathrm{T}} / \sigma=0.08$ is used to trim the blade in forward flight, and a value of $C_{p} / \sigma=0.0131$ is used to trim the blade in hover. The blade radius is 12.5 
feet, and the blade is discretized into 10 segments (NSEG $=10$ ). For the Min $\Sigma \beta$ approach 24 design variables are used (including the pseudo-design variables), and in the $\mathrm{K}-\mathrm{S}$ function approach 22 design variables are used.

Some results, obtained to date, are summarized in Table 1 and Figs 2 and 3. Table 1 presents a summary of preliminary optimization results. From Table 1 and Fig. 2 it can be seen that it is possible to obtain substantial increases in both the hover figure of merit, (21.7 - 28.8 percent), and the axial propulsive efficiency, $\eta_{a x}(24.6-41.3$ percent) using both multiobjective formulation techniques. It is of interest to note that the mean chord (and correspondingly the blade solidity) is increased by 71 percent and 40 percent in the $\mathrm{K}-\mathrm{S}$ function and Min $\Sigma \beta$ approaches, respectively from the baseline value. Two possible explanations exist for this large increases in the rotor solidity. First, in order to satisfy the frequency constraint, the root chord is significantly increased to make the stiffnesses larger, which in turn increases the solidity (see Fig. 3). Secondly, since the hover figure of merit is being maximized, $\sigma$ is being increased to increase the thrust margin of the rotor in hover.

Based on the previous experience, the above problem will be formulated with constraints on rotor solidity. The final paper will present results of the integrated aerodynamic/dynamic/aeroelastic optimization problem of high speed proprotors with additional design constraints. Design trade-off studies will also be performed by varying the flight conditions and the results of corresponding optimum blade designs will be presented. 
Table 1 Summary of Preliminary Optimization Results

\begin{tabular}{cccccc}
\hline & Reference & \multicolumn{2}{c}{ Bounds } & \multicolumn{2}{c}{ Optimum } \\
& blade & lower & upper & Min $\Sigma \beta$ & K-S \\
\hline \hline Objective & & & & & \\
Functions & & & & & \\
& & & & & \\
\hline FOM & 0.662 & - & - & 0.853 & 0.936 \\
$\eta_{\text {ax }}$ & 0.647 & - & - & 0.787 & 0.805 \\
\hline \hline Constraints & & & & & \\
\hline $\begin{array}{c}\text { W (lb) } \\
f_{1} \text { (per rev) }\end{array}$ & 0.812 & 1.00 & - & 1.01 & 1.34 \\
$\alpha_{1}$ & 0.096 & - & -0.001 & -0.040 & -1.529 \\
$\alpha_{2}$ & 0.096 & - & -0.001 & -0.040 & -1.529 \\
$\alpha_{3}$ & -0.697 & - & -0.001 & -0.732 & -0.169 \\
$\alpha_{4}$ & -0.697 & - & -0.001 & -0.732 & -0.169 \\
$\alpha_{5}$ & -2.431 & - & -0.001 & -2.443 & -2.502 \\
$\alpha_{6}$ & -0.170 & - & -0.001 & -0.265 & -0.073 \\
$\beta_{1}$ & 0.150 & 0.001 & 0.200 & 0.006 & - \\
$\beta_{2}$ & 0.150 & 0.001 & 0.200 & 0.010 & - \\
\hline \hline Mean chord \\
$c_{\mathrm{e}}$ (ft)
\end{tabular}




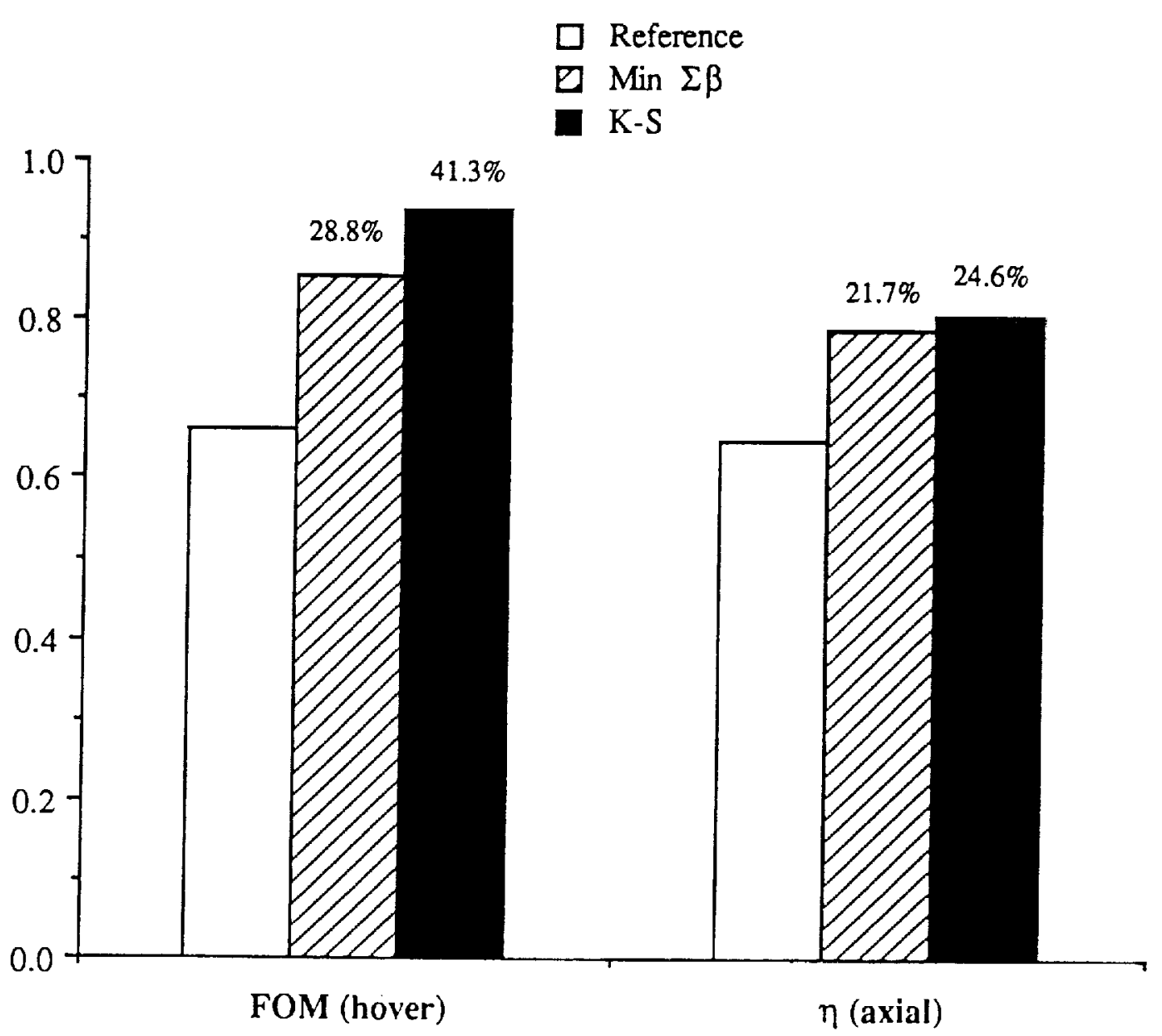

Figure 2 Comparison of individual objective functions 


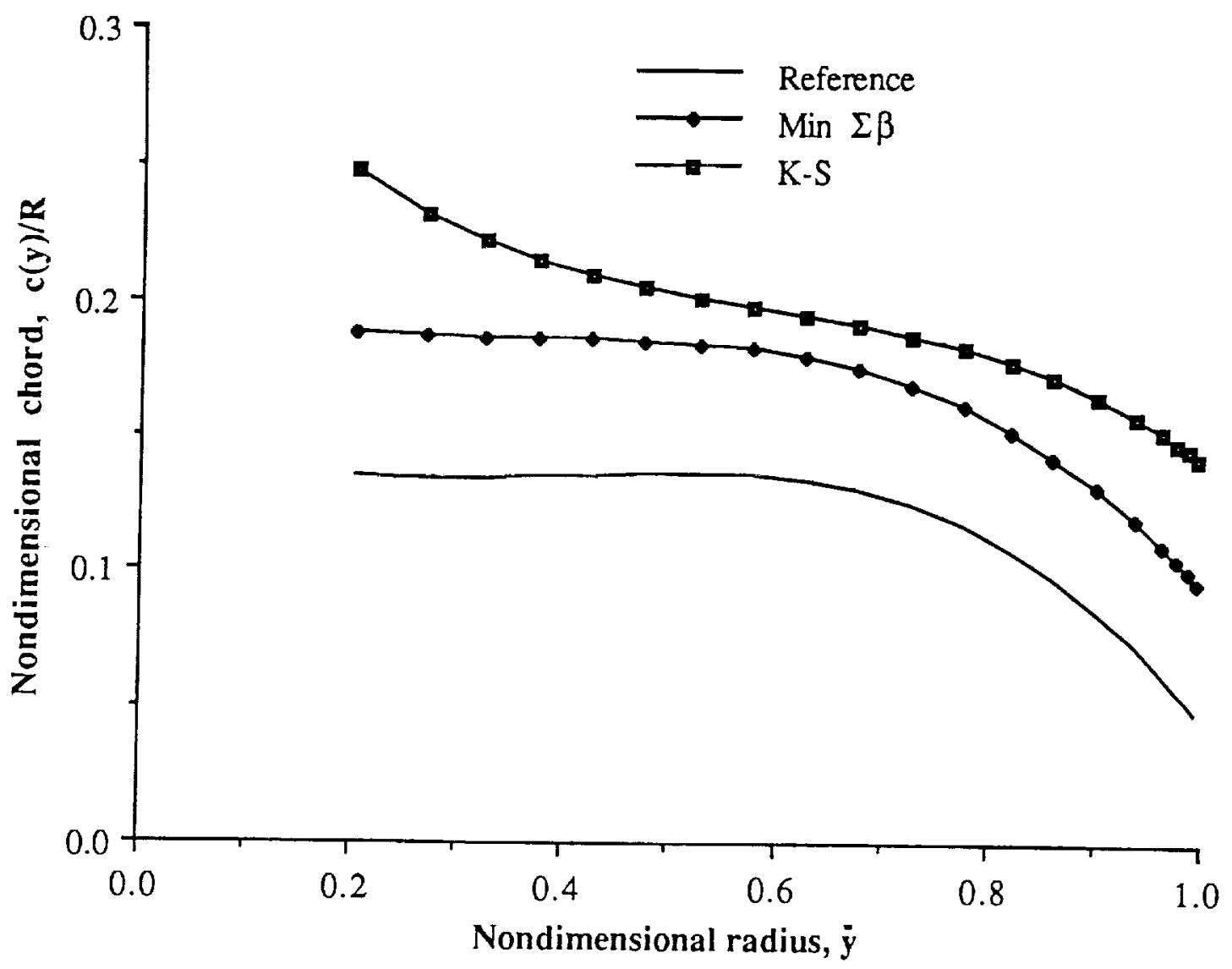

Figure 3 Chord distribution 


\section{Acknowledgements}

This work was accomplished under a grant from NASA Ames Research Center, Grant number NAG2-771, Technical Monitor, Mr. John Madden.

\section{References}

1. Talbot, P., Phillips, J. and Totah, J., "Selected Design Issues of Some High Speed Rotorcraft Concepts," presented at the AIAA/AHS/ASEE Aircraft Design, Sytems and Operations Conference, September 17-19, 1990, Dayton, Ohio.

2. Rutherford, J., O'Rourke, M., Martin, C., Lovenguth, M. and Mitchell, C., "Technology Needs for High Speed Rotorcraft," NASA CR 177578, NAS2 13070, April 1991.

3. Wilkerson, J. B., Schneider, J. J. and Bartie, K. M., "Technology Needs for High Speed Rotorcraft (1)," NASA CR 177585, NAS2 - 13041, May 1991.

4. Scott, M. W.. "Technology Needs for High Speed Rotorcraft (2)," NASA CR 177590, NAS2 - 13058, August 1991.

5. Detore, J. and Conway, S., "Technology Needs for High Speed Rotorcraft (3)," NASA CR XXXX, NAS2 - 13072, 1991.

6. Johnson, W., Lau, B. H. and Bowles, J. V., "Calculated Performance, Stability, and Maneuverability of High Speed Tilting Proprotor Aircraft," Vertica vol. 11, 1987.

7. Liu, J. and McVeigh, "Design of Swept Blade Rotors for High-Speed Tiltrotor Application," paper no. AIAA 91-3147 presented at the AIAA Aircraft Design Systems and Operations Meeting, September 23-25, 1991, Baltimore, Maryland

8. Chattopadhyay, A. and Narayan, J., "Optimum Design of High Speed Prop-Rotors Using a Multidisciplinary Approach," presented at the 48th Annual Forum of the American Helicopter Society, June 3-5, 1992, Washington D.C.

9. Chattopadhyay, A. and Narayan, J., "A Design Optimization Procedure for High-Speed Prop-Rotors," proc. 33rd AIAA/ASME/ASCE/AHS Structures, Structural Dynamics, and Materials Conference, Dallas, Texas, April 13-15, 1992. AIAA Paper No. 92-2375.

10. Weller, W. H. and Davis, M. W., "Application of Design Optimization Techniques to Rotor Dynamics Problems," Proc., 42nd Annual Forum of the AHS, Washington, D.C., June 2-4, 1986, pp. 27-44. 
11. Sobieszczanski-Sobieski, J., Dovi, A. and Wrenn, G., "A New Algorithm for General Multiobjective Optimization," NASA TM - 100536, March, 1988.

12. Wrenn, G. A., "An Indirect Method for Numerical Optimization Using the Kreisselmeier-Steinhauser Function," NASA CR - 4220, March 1989.

13. Johnson, W., "A Comprehensive Analytical Model of Rotorcraft Aerodynamics and Dynamics - Johnson Aeronautics Version," Vol. II. User's Manual.

14. Vanderplaats, G. N., "CONMIN - A Fortran Program for Constrained Function Minimization," User's Manual, NASA TMX-62282, August 1973.

15. Fadel, G. M., Riley, M. F. and Barthelemy, J. M., "Two Point Exponential Approximation method for Structural Optimization," Structural Optimization, Vol. 2., pp117-224. 